\title{
Multiple Bounded Positive Solutions to Integral Type BVPs for Singular Second Order ODEs on the Whole Line
}

\author{
Yuji Liu \\ Department of Mathematics, Guangdong University of Business Studies, Guangzhou 510320, China \\ Correspondence should be addressed to Yuji Liu, liuyuji888@sohu.com
}

Received 3 June 2012; Accepted 6 August 2012

Academic Editor: Ziemowit Popowicz

Copyright (C) 2012 Yuji Liu. This is an open access article distributed under the Creative Commons Attribution License, which permits unrestricted use, distribution, and reproduction in any medium, provided the original work is properly cited.

This paper is concerned with the integral type boundary value problems of the second order differential equations with one-dimensional $p$-Laplacian on the whole line. By constructing a suitable Banach space and a operator equation, sufficient conditions to guarantee the existence of at least three positive solutions of the BVPs are established. An example is presented to illustrate the main results. The emphasis is put on the one-dimensional $p$-Laplacian term $\left[\rho(t) \Phi\left(x^{\prime}(t)\right)\right]^{\prime}$ involved with the function $\rho$, which makes the solutions un-concave.

\section{Introduction}

The multipoint boundary-value problems for linear second order ordinary differential equations (ODEs for short) was initiated by Il'in and Moiseev [1]. Since then, more general nonlinear multi-point boundary-value problems (BVPs for short) were studied by several authors, see the text books [2-4] and the references cited therein.

Differential equations governed by nonlinear differential operators have been widely studied. In this setting the most investigated operator is the classical one-dimensional $p$ Laplacian, that is, $\Phi_{p}(x)=|x|^{p-2} x$ with $p>1$. This operator is involved in some models, for example, in non-Newtonian fluid theory, diffusion of flows in porous media, nonlinear elasticity, and theory of capillary surfaces. The related nonlinear differential equation has the form

$$
\left[\Phi\left(x^{\prime}\right)\right]^{\prime}=f\left(t, x, x^{\prime}\right), \quad t \in(-\infty,+\infty)
$$

where $\Phi(x)=|x|^{p-2} x$ with $p>1$ is a one dimensional $p$-Laplacian. For a comprehensive bibliography on this subject, see, for example [5-9]. 
In this paper, we consider the more generalized BVP for second order differential equation on the whole line with $p$-Laplacian coupled with the integral type BCs, that is the BVP

$$
\begin{gathered}
{\left[\rho(t) \Phi\left(x^{\prime}(t)\right)\right]^{\prime}+f\left(t, x(t), x^{\prime}(t)\right)=0, \quad t \in R,} \\
\lim _{t \rightarrow-\infty} x(t)=: x(-\infty)=\int_{-\infty}^{+\infty} g(s) x(s) d s, \\
\lim _{t \rightarrow+\infty} x(t)=: x(+\infty)=\int_{-\infty}^{+\infty} h(s) x(s) d s,
\end{gathered}
$$

where $f: R^{3} \rightarrow R$ is a nonnegative Caratheodory function, $g, h: R \rightarrow[0, \infty)$ satisfy $g, h \in L^{1}(R), \rho \in C(R,(0, \infty))$, the integrals in mentioned equations are meant in the sense of Riemann-Stieljes, $\Phi(x)=|x|^{p-2} x$ with $p>1$ is called a one dimensional $p$-Laplacian, whose inverse function is denoted by $\Phi^{-1}$.

The purpose is to establish sufficient conditions for the existence of at least three positive solutions of BVP(1.2). The result in this paper generalizes and improves some known ones since the one-dimensional $p$-Laplacian term $\left[\rho(t) \Phi\left(x^{\prime}(t)\right)\right]^{\prime}$ involved with the function $\rho$, which makes the solutions unconcave and there exists no paper concerned with the existence of at least three positive solutions of this kind of integral BVPs on the whole lines. This paper fills the gap.

The remainder of this paper is organized as follows: the main result (Theorem 2.8) is presented in Section 2, and the example to show the main result is given in Section 3.

\section{Main Results}

In this section, we first present some background definitions in Banach spaces and state an important three fixed point theorem. Then the main results are given and proved.

Definition 2.1. Let $X$ be a real Banach space. The nonempty convex closed subset $P$ of $X$ is called a cone in $X$ if $a x \in P$ for all $x \in P$ and $a \geq 0$ and $x \in X$ and $-x \in X$ imply $x=0$.

Definition 2.2. A map $\psi: P \rightarrow[0,+\infty)$ is a nonnegative continuous concave or convex functional map provided $\psi$ is nonnegative, continuous, and satisfies $\psi(t x+(1-t) y) \geq$ $t \psi(x)+(1-t) \psi(y)$, or $\psi(t x+(1-t) y) \leq t \psi(x)+(1-t) \psi(y)$, for all $x, y \in P$ and $t \in[0,1]$.

Definition 2.3. An operator $T: X \rightarrow X$ is completely continuous if it is continuous and maps bounded sets into precompact sets.

Definition 2.4. Let $a, b, c, d, h>0$ be positive constants, $\alpha, \psi$ be two nonnegative continuous concave functionals on the cone $P, \gamma, \beta, \theta$ be three nonnegative continuous convex functionals on the cone $P$. Define the convex sets as follows:

$$
\begin{gathered}
P_{c}=\{x \in P:\|x\|<c\}, \\
P(\gamma, \alpha ; a, c)=\{x \in P: \alpha(x) \geq a, \gamma(x) \leq c\}, \\
P(\gamma, \theta, \alpha ; a, b, c)=\{x \in P: \alpha(x) \geq a, \theta(x) \leq b, \gamma(x) \leq c\}, \\
Q(\gamma, \beta ;, d, c)=\{x \in P: \beta(x) \leq d, \gamma(x) \leq c\}, \\
Q(\gamma, \beta, \psi ; h, d, c)=\{x \in P: \psi(x) \geq h, \beta(x) \leq d, \gamma(x) \leq c\} .
\end{gathered}
$$


Lemma 2.5 (see [10]). Let $X$ be a real Banach space, $P$ be a cone in $X, \alpha, \psi$ be two nonnegative continuous concave functionals on the cone $P, \gamma, \beta, \theta$ be three nonnegative continuous convex functionals on the cone P. Assume that there exists a constant $M>0$ such that

$$
\alpha(x) \leq \beta(x), \quad\|x\| \leq M \gamma(x), \quad \forall x \in P .
$$

Furthermore, suppose that $h, d, a, b, c>0$ are constants with $d<$ a. Let $T: \overline{P_{c}} \rightarrow \overline{P_{c}}$ be a completely continuous operator. If

(C1) $\{x \in P(\gamma, \theta, \alpha ; a, b, c) \mid \alpha(x)>a\} \neq \emptyset$ and $\alpha(T x)>$ a for every $x \in P(\gamma, \theta, \alpha ; a, b, c)$;

(C2) $\{x \in Q(\gamma, \theta, \psi ; h, d, c) \mid \beta(x)<d\} \neq \emptyset$ and $\beta(T x)<d$ for every $x \in Q(\gamma, \theta, \psi ; h, d, c)$;

(C3) $\alpha(T x)>$ a for $x \in P(\gamma, \alpha ; a, c)$ with $\theta(T x)>b$;

(C4) $\beta(T x)<d$ for each $x \in Q(\gamma, \beta ; d, c)$ with $\psi(T x)<h$,

then $T$ has at least three fixed points $x_{1}, x_{2}$ and $x_{3}$ such that $\beta\left(x_{1}\right)<d, \alpha\left(x_{2}\right)>a, \beta\left(x_{3}\right)>$ $d, \alpha\left(x_{3}\right)<a$.

Let us list the assumptions

$(\mathrm{H} 1) g, h: R \rightarrow[0, \infty)$ satisfy $\int_{-\infty}^{+\infty} g(s) d s<1, \int_{-\infty}^{+\infty} h(s) d s<1$ and

$$
g(t)\left(1-\int_{-\infty}^{+\infty} h(s) d s\right)-h(t)\left(1-\int_{-\infty}^{+\infty} g(s) d s\right) \geq 0, \quad t \in R
$$

(H2) $\rho \in C(R), \rho(t)>0$ for $t \in R$ with $\int_{-\infty}^{0} \Phi^{-1}(1 / \rho(t)) d t=\int_{0}^{+\infty} \Phi^{-1}(1 / \rho(t)) d t<+\infty$.

(H3) $f(t, c, 0) \not \equiv$ on any finite subinterval of $R$ for each $c \in R, f: R^{3} \rightarrow R$ is a Carathédory function, that is,

(i) $t \rightarrow f\left(t, x,\left(1 / \Phi^{-1}(\rho(t))\right) y\right)$ is measurable for any $(x, y) \in R^{2}$,

(ii) $(x, y) \rightarrow f\left(t, x,\left(1 / \Phi^{-1}(\rho(t))\right) y\right)$ is continuous for a.e. $t \in R$,

(iii) for each $r>0$, there exists nonnegative function $\phi_{r} \in L^{1}(R)$ such that $\max \{|u|,|v|\} \leq$ $r$ implies

$$
\left|f\left(t, u, \frac{1}{\Phi^{-1}(\rho(t))} v\right)\right| \leq \phi_{r}(t), \quad \text { a.e. } t \in R .
$$

Choose

$$
X=\left\{x \in C^{1}(R): \begin{array}{cc} 
& \text { and there exist the limits } \\
& \lim _{t \rightarrow-\infty} x(t), \\
& \lim _{t \rightarrow+\infty} x(t), \\
& \lim _{t \rightarrow-\infty} \Phi^{-1}(\rho(t)) x^{\prime}(t) \\
\text { and } \lim _{t \rightarrow+\infty} \Phi^{-1}(\rho(t)) x^{\prime}(t)
\end{array}\right\} .
$$


For $x \in X$, define the norm of $x$ by

$$
\|x\|=\max \left\{\sup _{t \in R}|x(t)|, \sup _{t \in R} \Phi^{-1}(\rho(t)) x^{\prime}(t)\right\}
$$

One can prove that $X$ is a Banach space with the norm $\|x\|$ for $x \in X$.

Let $x \in X$. Consider the following auxiliary BVP

$$
\begin{gathered}
{\left[\rho(t) \Phi\left(y^{\prime}(t)\right)\right]^{\prime}+f\left(t, x(t), x^{\prime}(t)\right)=0, \quad t \in R,} \\
y(-\infty)=\int_{-\infty}^{+\infty} g(s) y(s) d s \\
y(+\infty)=\int_{-\infty}^{+\infty} h(s) y(s) d s .
\end{gathered}
$$

Lemma 2.6. Suppose that (H1)-(H3) hold. If $y \in C^{1}(R)$ such that $\left[\rho \Phi\left(y^{\prime}\right)\right]^{\prime} \in L^{1}(R)$ is a solution of $B V P(2.7)$, then

(i) $y$ is bounded and nonnegative on $R$;

(ii) $y(t)$ is concave with respect to $\tau=\int_{-\infty}^{t} \Phi^{-1}(1 / \rho(s)) d s / \int_{-\infty}^{+\infty} \Phi^{-1}(1 / \rho(s)) d s$;

(iii) for $k>0$, it holds that $\min _{t \in[-k, k]} y(t) \geq \mu \sup _{t \in R} y(t)$ with $\mu=\int_{-\infty}^{-k} \Phi^{-1}(1 / \rho(s)) d s / 2$ $\int_{-\infty}^{+\infty} \Phi^{-1}(1 / \rho(s)) d s ;$

(iv) there exists a unique constant $A_{x} \in\left[-\int_{-\infty}^{+\infty} f\left(s, x(s), x^{\prime}(s)\right) d s, 0\right]$ such that

$$
\begin{aligned}
& \int_{-\infty}^{+\infty} \frac{g(t)\left(1-\int_{-\infty}^{+\infty} h(s) d s\right)-h(t)\left(1-\int_{-\infty}^{+\infty} g(s) d s\right)}{1-\int_{-\infty}^{+\infty} g(s) d s} \\
& \quad \times \int_{-\infty}^{t} \Phi^{-1}\left(\frac{1}{\rho(s)}\right) \Phi^{-1}\left(A_{x}+\int_{s}^{+\infty} f\left(u, x(u), x^{\prime}(u)\right) d u\right) d s d t \\
& \quad+\int_{-\infty}^{+\infty} \Phi^{-1}\left(\frac{1}{\rho(s)}\right) \Phi^{-1}\left(A_{x}+\int_{s}^{+\infty} f\left(u, x(u), x^{\prime}(u)\right) d u\right) d s=0 .
\end{aligned}
$$

Proof. Since $x \in X$, we get

$$
r=\max \left\{\sup _{t \in R}|x(t)|, \sup _{t \in R} \Phi^{-1}(\rho(t)) x^{\prime}(t)\right\}<+\infty .
$$

Then there exists a nonnegative function $\phi_{r} \in L^{1}(-\infty,+\infty)$ such that

$$
0 \leq f\left(t, x(t), x^{\prime}(t)\right)=f\left(t, x(t), \frac{1}{\Phi^{-1}(\rho(t))} \Phi^{-1}(\rho(t)) x^{\prime}(t)\right) \leq \phi_{r}(t), \quad t \in R .
$$


Then

$$
\int_{-\infty}^{+\infty} f\left(s, x(s), x^{\prime}(s)\right) d s \text { is convergent. }
$$

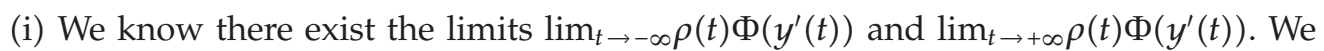
claim that there exists $\tau_{0} \in R$ such that $y^{\prime}\left(\tau_{0}\right)=0$. In fact, if $y^{\prime}(t)>0$ for all $t \in R$, we get $y(-\infty)<y(t)<y(+\infty)$ for all $t \in R$. It follows that

$$
y(-\infty) \geq \int_{-\infty}^{+\infty} g(s) d s y(-\infty), \quad y(+\infty) \leq \int_{-\infty}^{+\infty} h(s) d s y(+\infty)
$$

Then (H1) implies $y(-\infty) \geq 0 \geq y(+\infty)$, which contradicts to $y^{\prime}(t)>0$ for all $t \in R$. Similarly we can prove that $y^{\prime}(t)<0$ does not hold. Then there exists $\tau_{0} \in R$ such that $y^{\prime}\left(\tau_{0}\right)=0$.

Since $\left[\rho(t) \Phi\left(y^{\prime}(t)\right)\right]^{\prime}=-f\left(t, x(t), x^{\prime}(t)\right) \leq 0$, we know that $\rho(t) \Phi\left(y^{\prime}(t)\right)$ is decreasing on $R$. Then $\rho(t) \Phi\left(y^{\prime}(t)\right) \geq 0$ for all $t \leq \tau_{0}$ and $\rho(t) \Phi\left(y^{\prime}(t)\right) \leq 0$ for all $t \geq \tau_{0}$. Hence

$$
y \text { is increasing for } t \in\left(-\infty, \tau_{0}\right] \text { and decreasing for } t \in\left[\tau_{0},+\infty\right) \text {. }
$$

One sees that

$$
y^{\prime}(t)= \begin{cases}-\Phi^{-1}\left(\frac{1}{\rho(t)} \int_{\tau_{0}}^{t} f\left(u, x(u), x^{\prime}(u)\right) d u\right)=:-G(t), & t \geq \tau_{0} \\ \Phi^{-1}\left(\frac{1}{\rho(t)} \int_{t}^{\tau_{0}} f\left(u, x(u), x^{\prime}(u)\right) d u\right)=: H(t), & t \leq \tau_{0} .\end{cases}
$$

Since $\int_{-\infty}^{+\infty} \Phi^{-1}(1 / \rho(t)) d t<+\infty$ and $\int_{-\infty}^{+\infty} f\left(t, x(t), x^{\prime}(t)\right) d t<+\infty$, we see that

$$
\text { both } \int_{t}^{+\infty} G(s) d s, \int_{-\infty}^{t} H(s) d s \text { are convergent. }
$$

Then we get that

$$
y(t)= \begin{cases}y(+\infty)+\int_{t}^{+\infty} G(s) d s, & t \geq \tau_{0}, \\ y(-\infty)+\int_{-\infty}^{t} H(s) d s, & t \leq \tau_{0} .\end{cases}
$$

This tells us that $y$ is bounded on $R$. 
It follows from (2.7) and (2.16) that

$$
\begin{aligned}
y(-\infty)= & \int_{-\infty}^{\tau_{0}} g(s) d s y(-\infty)+\int_{\tau_{0}}^{+\infty} g(s) d s y(+\infty)+\int_{-\infty}^{\tau_{0}} g(t) \int_{-\infty}^{t} H(s) d s d t \\
& +\int_{\tau_{0}}^{+\infty} g(t) \int_{t}^{+\infty} G(s) d s d t \\
y(+\infty)= & \int_{-\infty}^{\tau_{0}} h(s) d s y(-\infty)+\int_{\tau_{0}}^{+\infty} h(s) d s y(+\infty)+\int_{-\infty}^{\tau_{0}} h(t) \int_{-\infty}^{t} H(s) d s d t \\
& +\int_{\tau_{0}}^{+\infty} h(t) \int_{t}^{+\infty} G(s) d s d t .
\end{aligned}
$$

Then

$$
\begin{aligned}
(1- & \left.\int_{-\infty}^{\tau_{0}} g(s) d s\right) y(-\infty)-\int_{\tau_{0}}^{+\infty} g(s) d s y(+\infty) \\
= & \int_{-\infty}^{\tau_{0}} g(t) \int_{-\infty}^{t} t H(s) d s d t+\int_{\tau_{0}}^{+\infty} g(t) \int_{t}^{+\infty} G(s) d s d t \\
& -\int_{-\infty}^{\tau_{0}} h(s) d s y(-\infty)+\left(1-\int_{\tau_{0}}^{+\infty} h(s) d s\right) y(+\infty) \\
= & \int_{-\infty}^{\tau_{0}} h(t) \int_{-\infty}^{t} H(s) d s d t+\int_{\tau_{0}}^{+\infty} h(t) \int_{t}^{+\infty} G(s) d s d t .
\end{aligned}
$$

Hence

$$
\begin{aligned}
& y(-\infty)=\frac{\left|\begin{array}{ll}
\int_{-\infty}^{\tau_{0}} g(t) \int_{-\infty}^{t} H(s) d s d t+\int_{\tau_{0}}^{+\infty} g(t) \int_{t}^{+\infty} G(s) d s d t-\int_{\tau_{0}}^{+\infty} g(s) d s \\
\int_{-\infty}^{\tau_{0}} h(t) \int_{-\infty}^{t} H(s) d s d t+\int_{\tau_{0}}^{+\infty} h(t) \int_{t}^{+\infty} G(s) d s d t 1-\int_{\tau_{0}}^{+\infty} h(s) d s
\end{array}\right|}{\left|\begin{array}{ll}
1-\int_{-\infty}^{\tau_{0}} g(s) d s & -\int_{\tau_{0}}^{+\infty} g(s) d s \\
-\int_{-\infty}^{\tau_{0}} h(s) d s & 1-\int_{\tau_{0}}^{+\infty} h(s) d s
\end{array}\right|},
\end{aligned}
$$

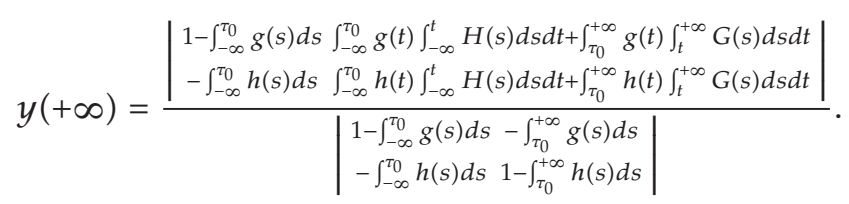

Since

$$
\left|\begin{array}{cc}
1-\int_{-\infty}^{\tau_{0}} g(s) d s & -\int_{\tau_{0}}^{+\infty} g(s) d s \\
-\int_{-\infty}^{\tau_{0}} h(s) d s & 1-\int_{\tau_{0}}^{+\infty} h(s) d s
\end{array}\right|=\left|\begin{array}{cc}
1-\int_{-\infty}^{+\infty} g(s) d s & -\int_{\tau_{0}}^{+\infty} g(s) d s \\
1-\int_{-\infty}^{+\infty} h(s) d s & 1-\int_{\tau_{0}}^{+\infty} h(s) d s
\end{array}\right|>0,
$$


we get from (2.19) that $y(-\infty) \geq 0$ and $y(+\infty) \geq 0$. Hence (2.16) implies that

$$
y(t) \geq 0, \quad \forall t \in R
$$

(ii) We prove that $y(t)$ is concave with respect to $\tau$ on $R$. It is easy to see that $\tau \in$ $C(R,(0,1))$ and

$$
\frac{d \tau}{d t}=\Phi^{-1}\left(\frac{1}{\rho(t)}\right) \frac{1}{\int_{-\infty}^{+\infty} \Phi^{-1}(1 / \rho(s)) d s}>0
$$

Thus

$$
\frac{d y}{d t}=\frac{d y}{d \tau} \frac{d \tau}{d t}=\frac{d y}{d \tau} \Phi^{-1}\left(\frac{1}{\rho(t)}\right) \frac{1}{\int_{-\infty}^{+\infty} \Phi^{-1}(1 / \rho(s)) d s}
$$

It follows that

$$
\rho(t) \Phi\left(\frac{d y}{d t}\right)=\Phi\left(\frac{d y}{d \tau}\right) \Phi\left(\frac{1}{\int_{-\infty}^{+\infty} \Phi^{-1}(1 / \rho(s)) d s}\right) .
$$

Hence

$$
\left[\rho(t) \Phi\left(\frac{d y}{d t}\right)\right]^{\prime}=\Phi^{\prime}\left(\frac{d y}{d \tau}\right) \frac{d^{2} y}{d \tau^{2}} \frac{d \tau}{d t} \Phi\left(\frac{1}{\int_{-\infty}^{+\infty} \Phi^{-1}(1 / \rho(s)) d s}\right) .
$$

So

$$
\frac{d^{2} y}{d \tau^{2}}=\Phi\left(\int_{-\infty}^{+\infty} \Phi^{-1}\left(\frac{1}{\rho(s)}\right) d s\right) \frac{[\rho(t) \Phi(d y / d t)]^{\prime}}{\Phi^{\prime}(d y / d \tau)(d \tau / d t)}
$$

Since $\left[\rho(t) \Phi\left(y^{\prime}(t)\right)\right]^{\prime} \leq 0, \Phi^{\prime}(y)>0(y \neq 0)$ and $(d \tau / d t)>0$, we get that $\left(d^{2} y / d \tau^{2}\right) \leq 0$. Hence $y(t)$ is concave with respect to $\tau$ on $R$.

(iii) Now, we prove that

$$
\min _{t \in[-k, k]} y(t) \geq \mu \sup _{t \in R} y(t)
$$

Since $d \tau / d t>0$ for all $t \in R$, there exists the inverse function of $\tau=\tau(t)$. Denote the inverse function of $\tau=\tau(t)$ by $t=t(\tau)$.

It follows from (2.13) that $\sup _{t \in R} y(t)=y\left(\tau_{0}\right)$. One sees

$$
\min _{t \in[-k, k]} y(t)=\min \{y(-k), y(k)\}
$$


If $\min \{y(-k), y(k)\}=y(k)=y(t(\tau(k)))$, note $\tau(k)<1$, then for $t \in[-k, k]$, one has

$$
y(t) \geq y(t(\tau(k)))=y\left(t\left(\frac{1-\tau(k)+\tau\left(\tau_{0}\right)}{1+\tau\left(\tau_{0}\right)} \frac{\tau(k)}{1-\tau(k)+\tau\left(\tau_{0}\right)}+\frac{\tau(k)}{1+\tau\left(\tau_{0}\right)} \tau\left(\tau_{0}\right)\right)\right) .
$$

Noting that $1>\tau(k)$ and $y(t)$ is concave with respect to $\tau$, then, for $t \in[-k, k]$,

$$
\begin{aligned}
y(t) & \geq \frac{1-\tau(k)+\tau\left(\tau_{0}\right)}{1+\tau\left(\tau_{0}\right)} y\left(t\left(\frac{\tau(k)}{1-\tau(k)+\tau\left(\tau_{0}\right)}\right)\right)+\frac{\tau(k)}{1+\tau\left(\tau_{0}\right)} y\left(t\left(\tau\left(\tau_{0}\right)\right)\right) \\
& \geq \int_{-\infty}^{k} \Phi^{-1}\left(\frac{1}{\rho(s)}\right) d s \frac{1}{2 \int_{-\infty}^{+\infty} \Phi^{-1}(1 / \rho(s)) d s} y\left(\tau_{0}\right) \\
& =\mu \sup _{t \in R} y(t) .
\end{aligned}
$$

Similarly, if $\min \{y(-k), y(k)\}=y(-k)=(y(t(\tau(-k)))$, note $\tau(-k)<1$, for $t \in[-k, k]$, one has

$$
\begin{aligned}
y(t) & \geq y(t(\tau(-k))) \\
& =y\left(t\left(\frac{1+\tau\left(\tau_{0}\right)-\tau(-k)}{1+\tau\left(\tau_{0}\right)} \frac{\tau(-k)}{1+\tau\left(\tau_{0}\right)-\tau(-k)}+\frac{\tau(-k)}{1+\tau\left(\tau_{0}\right)} \tau\left(\tau_{0}\right)\right)\right) \\
& \geq \frac{1+\tau\left(\tau_{0}\right)-\tau(-k)}{1+\tau\left(\tau_{0}\right)} y\left(t\left(\frac{\tau(-k)}{1+\tau\left(\tau_{0}\right)-\tau(-k)}\right)\right)+\frac{\tau(-k)}{1+\tau\left(\tau_{0}\right)} y\left(t\left(\tau\left(\tau_{0}\right)\right)\right) \\
& \geq \int_{-\infty}^{-k} \Phi^{-1}\left(\frac{1}{\rho(s)}\right) d s \frac{1}{2 \int_{-\infty}^{+\infty} \Phi^{-1}(1 / \rho(s)) d s} y\left(\tau_{0}\right) \\
& >\mu \sup _{t \in R} y(t) .
\end{aligned}
$$

Hence (2.27) holds.

(iv) Finally, we prove the uniqueness of $A_{x}$. Define

$$
\begin{aligned}
H_{x}(c)= & \int_{-\infty}^{+\infty} \frac{g(t)\left(1-\int_{-\infty}^{+\infty} h(s) d s\right)-h(t)\left(1-\int_{-\infty}^{+\infty} g(s) d s\right)}{1-\int_{-\infty}^{+\infty} g(s) d s} \\
& \times \int_{-\infty}^{t} \Phi^{-1}\left(\frac{1}{\rho(s)}\right) \Phi^{-1}\left(c+\int_{s}^{+\infty} f\left(u, x(u), x^{\prime}(u)\right) d u\right) d s d t \\
& +\int_{-\infty}^{+\infty} \Phi^{-1}\left(\frac{1}{\rho(s)}\right) \Phi^{-1}\left(c+\int_{s}^{+\infty} f\left(u, x(u), x^{\prime}(u)\right) d u\right) d s .
\end{aligned}
$$

Then (H1) implies that $H_{x} \in C(R, R)$ is increasing on $R$ and $H_{x}(0) \geq 0$. Let

$$
\bar{c}=-\int_{-\infty}^{+\infty} f\left(u, x(u), x^{\prime}(u)\right) d u,
$$


then $H_{x}(\bar{c}) \leq 0$. By mean value theorem, there exists an unique $A_{x} \in[\bar{c}, 0]$ satisfying $H_{x}\left(A_{x}\right)=0$. Then (iv) holds. This completes the proof of the lemma.

Choose $1 \geq k>0$ and

$$
\mu=\frac{\int_{-\infty}^{-k} \Phi^{-1}(1 / \rho(s)) d s}{2 \int_{-\infty}^{\infty} \Phi^{-1}(1 / \rho(s)) d s} .
$$

Define the cone $P \subseteq X$ by

$$
P=\left\{x \in X: \begin{array}{c}
x(t) \geq 0, t \in R, \\
\min _{t \in[-k, k]} x(t) \geq \mu \max _{t \in R} x(t)
\end{array}\right\} .
$$

It is easy to see that $P$ is a cone in $X$.

Define the operator $T: P \rightarrow X$ by

$$
\begin{aligned}
(T x)(t)= & \frac{\int_{-\infty}^{+\infty} g(u) \int_{-\infty}^{u} \Phi^{-1}(1 / \rho(s)) \Phi^{-1}\left(A_{x}+\int_{s}^{+\infty} f\left(r, x(r), x^{\prime}(r)\right) d r\right) d s d u}{1-\int_{-\infty}^{+\infty} g(s) d s} \\
& +\int_{-\infty}^{t} \Phi^{-1} \frac{1}{\rho(s)} \Phi^{-1}\left(A_{x}+\int_{s}^{+\infty} f\left(r, x(r), x^{\prime}(r)\right) d r\right) d s
\end{aligned}
$$

where $A_{x}$ satisfies

$$
\begin{aligned}
& \int_{-\infty}^{+\infty} \frac{g(u)\left(1-\int_{-\infty}^{+\infty} h(s) d s\right)-h(u)\left(1-\int_{-\infty}^{+\infty} g(s) d s\right)}{1-\int_{-\infty}^{+\infty} g(s) d s} \\
& \quad \times \int_{-\infty}^{u} \Phi^{-1}\left(\frac{1}{\rho(s)}\right) \Phi^{-1}\left(A_{x}+\int_{s}^{+\infty} f\left(r, x(r), x^{\prime}(r)\right) d r\right) d s d u \\
& \quad+\int_{-\infty}^{+\infty} \Phi^{-1}\left(\frac{1}{\rho(s)}\right) \Phi^{-1}\left(A_{x}+\int_{s}^{+\infty} f\left(r, x(r), x^{\prime}(r)\right) d r\right) d s=0 .
\end{aligned}
$$

It follows from Lemma 2.6(iv) that $T: P \rightarrow X$ is well defined and $A_{x} \in\left[-\int_{-\infty}^{+\infty} f(s, x(s)\right.$, $\left.\left.x^{\prime}(s)\right) d s, 0\right]$. It is easy to show that

$$
\begin{gathered}
{\left[\rho(t) \Phi\left((T x)^{\prime}(t)\right)\right]^{\prime}+f\left(t, x(t) x^{\prime}(t)\right)=0, \quad t \in R,} \\
(T x)(-\infty)=\int_{-\infty}^{+\infty} g(s)(T x)(s) d s, \\
(T x)(+\infty)=\int_{-\infty}^{+\infty} h(s)(T x)(s) d s .
\end{gathered}
$$

It follows from Lemma 2.6(i) and (iii) that $T x \in P$ for all $x \in P$. Then $T: P \rightarrow P$ is well defined. 
Lemma 2.7. Suppose (H1)-(H3) hold. Then $T$ is completely continuous.

Proof. First, we prove that $T$ is continuous.

We claim that the function $A_{x}: P \rightarrow[0,+\infty)$ is continuous in $x$.

Let $\left\{x_{n}\right\} \in P$ with $x_{n} \rightarrow x_{0} \in P$ as $n \rightarrow \infty$ in $P$. Let $\left\{A_{x_{n}}\right\}(n=1,2, \ldots)$ be the constants determined by

$$
\begin{aligned}
& \int_{-\infty}^{+\infty} \frac{g(t)\left(1-\int_{-\infty}^{+\infty} h(s) d s\right)-h(t)\left(1-\int_{-\infty}^{+\infty} g(s) d s\right)}{1-\int_{-\infty}^{+\infty} g(s) d s} \\
& \quad \times \int_{-\infty}^{t} \Phi^{-1}\left(\frac{1}{\rho(s)}\right) \Phi^{-1}\left(A_{x_{n}}+\int_{s}^{+\infty} f\left(r, x_{n}(r), x_{n}^{\prime}(r)\right) d r\right) d s d t \\
& \quad+\int_{-\infty}^{+\infty} \Phi^{-1}\left(\frac{1}{\rho(s)}\right) \Phi^{-1}\left(A_{x_{n}}+\int_{s}^{+\infty} f\left(r, x_{n}(r), x_{n}^{\prime}(r)\right) d r\right) d s=0
\end{aligned}
$$

Since $x_{n} \rightarrow x_{0}$ in $P$ as $n \rightarrow \infty$, there exists an $r>0$ such that $\left\|x_{n}\right\| \leq r$. The fact $f$ is a Carathédory function means that there exists a nonnegative function $\phi_{r} \in L^{1}(-\infty,+\infty)$ such that

$$
0 \leq f\left(t, x_{n}(t), x_{n}^{\prime}(t)\right)=f\left(t, x_{n}(t), \frac{1}{\Phi^{-1}(\rho(t))} \Phi^{-1}(\rho(t)) x_{n}^{\prime}(t)\right) \leq \phi_{r}(t), \quad t \in R .
$$

Then

$$
\int_{-\infty}^{+\infty} f\left(s, x_{n}(s), x_{n}^{\prime}(s)\right) d s \text { is convergent. }
$$

So

$$
A_{x_{n}} \in\left[-\int_{-\infty}^{+\infty} f\left(s, x_{n}(s), x_{n}^{\prime}(s)\right) d s, 0\right] \subseteq\left[-\int_{-\infty}^{+\infty} \phi_{r}(s) d s, 0\right],
$$

which means that $\left\{A_{x_{n}}\right\}$ is uniformly bounded.

Suppose that $\left\{A_{x_{n}}\right\}$ does not converge to $A_{x_{0}}$. By the bounded property, we know that there exist two subsequences $\left\{A_{x_{n_{k}}}^{(1)}\right\}$ and $\left\{A_{x_{n_{k}}}^{(2)}\right\}$ of $\left\{A_{x_{n_{k}}}\right\}$ with $A_{x_{n_{k}}}^{(1)} \rightarrow c_{1}$ and $A_{x_{n_{k}}}^{(2)} \rightarrow c_{2}$ and $c_{1} \neq c_{2}$. 
By the construction of $A_{x_{n}},(n=1,2, \ldots)$, we have

$$
\begin{aligned}
& \int_{-\infty}^{+\infty} \frac{g(t)\left(1-\int_{-\infty}^{+\infty} h(s) d s\right)-h(t)\left(1-\int_{-\infty}^{+\infty} g(s) d s\right)}{1-\int_{-\infty}^{+\infty} g(s) d s} \\
& \quad \times \int_{-\infty}^{t} \Phi^{-1}\left(\frac{1}{\rho(s)}\right) \Phi^{-1}\left(A_{x_{n_{k}}}^{(1)}+\int_{s}^{+\infty} f\left(u, x_{n_{k}}(u), x_{n_{k}}^{\prime}(u)\right) d u\right) d s d t \\
& \quad+\int_{-\infty}^{+\infty} \Phi^{-1}\left(\frac{1}{\rho(s)}\right) \Phi^{-1}\left(A_{x_{n_{k}}}^{(1)}+\int_{s}^{+\infty} f\left(u, x_{n_{k}}(u), x_{n_{k}}^{\prime}(u)\right) d u\right) d s=0 .
\end{aligned}
$$

Let $k \rightarrow \infty$, using Lebesgue's dominated convergence theorem, the above equality implies

$$
\begin{aligned}
& \int_{-\infty}^{+\infty} \frac{g(t)\left(1-\int_{-\infty}^{+\infty} h(s) d s\right)-h(t)\left(1-\int_{-\infty}^{+\infty} g(s) d s\right)}{1-\int_{-\infty}^{+\infty} g(s) d s} \\
& \quad \times \int_{-\infty}^{t} \Phi^{-1}\left(\frac{1}{\rho(s)}\right) \Phi^{-1}\left(c_{1}+\int_{s}^{+\infty} f\left(u, x_{0}(u), x_{o}^{\prime}(u)\right) d u\right) d s d t \\
& \quad+\int_{-\infty}^{+\infty} \Phi^{-1}\left(\frac{1}{\rho(s)}\right) \Phi^{-1}\left(c_{1}+\int_{s}^{+\infty} f\left(u, x_{0}(u), x_{o}^{\prime}(u)\right) d u\right) d s=0 .
\end{aligned}
$$

By Lemma 2.6 (iv), we get $c_{1}=A_{x_{0}}$. Similarly, $c_{2}=A_{x_{0}}$. Thus $c_{1}=c_{2}$, a contradiction. So, for any $x_{n} \rightarrow x_{0}$, one has $A_{x_{n}} \rightarrow A_{x_{0}}$, which means $A_{x}: P \rightarrow R$ is continuous.

Since $A_{x}$ is continuous, together with the continuity of $(x, y) \rightarrow f\left(t, x,\left(1 / \Phi^{-1}(\rho\right.\right.$ $(t))) y$ ), we get that $T$ is continuous.

Second, we show that $T$ is maps bounded subsets into bounded sets.

Let $D \subseteq P$ be bounded. Then, there exists $r>0$ such that $D \subseteq\{x \in P:\|x\| \leq r\}$. Hence

$$
r=\max \left\{\sup _{t \in R}|x(t)|, \sup _{t \in R} \Phi^{-1}(\rho(t)) x^{\prime}(t)\right\}<+\infty
$$

Then there exists a nonnegative function $\phi_{r} \in L^{1}(-\infty,+\infty)$ such that

$$
0 \leq f\left(t, x(t), x^{\prime}(t)\right)=f\left(t, x(t), \frac{1}{\Phi^{-1}(\rho(t))} \Phi^{-1}(\rho(t)) x^{\prime}(t)\right) \leq \phi_{r}(t), \quad t \in R
$$

Then

$$
\int_{-\infty}^{+\infty} f\left(t, x(t), x^{\prime}(t)\right) d t \leq \int_{-\infty}^{+\infty} \phi_{r}(t) d t:=L
$$


Thus $\left|A_{x}\right| \leq L$ for all $x \in D$. Therefore,

$$
\begin{aligned}
(T x)(t)= & \frac{\int_{-\infty}^{+\infty} g(t) \int_{-\infty}^{t} \Phi^{-1}(1 / \rho(s)) \Phi^{-1}\left(A_{x}+\int_{s}^{+\infty} f\left(u, x(u), x^{\prime}(u)\right) d u\right) d s d t}{1-\int_{-\infty}^{+\infty} g(s) d s} \\
& +\int_{-\infty}^{t} \Phi^{-1}\left(\frac{1}{\rho(s)}\right) \Phi^{-1}\left(A_{x}+\int_{s}^{+\infty} f\left(u, x(u), x^{\prime}(u)\right) d u\right) d s \\
\leq & \left(\frac{\int_{-\infty}^{+\infty} g(t) \int_{-\infty}^{t} \Phi^{-1}(1 / \rho(s)) d s d t}{1-\int_{-\infty}^{+\infty} g(s) d s}+\int_{-\infty}^{t} \Phi^{-1}\left(\frac{1}{\rho(s)}\right) d s\right) \Phi^{-1}(2 L) \\
\leq & \left(\frac{\int_{-\infty}^{+\infty} g(t) \int_{-\infty}^{t} \Phi^{-1}(1 / \rho(s)) d s d t}{1-\int_{-\infty}^{+\infty} g(s) d s}+\int_{-\infty}^{+\infty} \Phi^{-1}\left(\frac{1}{\rho(s)}\right) d s\right) \Phi^{-1}(2 L)=: M_{1} .
\end{aligned}
$$

On the other hand, we have

$$
\Phi^{-1}(\rho(t))\left|(T x)^{\prime}(t)\right|=\left|\Phi^{-1}\left(A_{x}+\int_{t}^{\infty} f\left(u, x(u), x^{\prime}(u)\right) d u\right)\right| \leq \Phi^{-1}(2 L)=: M_{2} .
$$

Then

$$
\|(T x)\|=\max \left\{\sup _{t \in R}(T x)(t), \sup _{t \in R} \Phi^{-1}(\rho(t))(T x)^{\prime}(t)\right\}<\infty .
$$

So, $\{T x: x \in D\}$ is bounded.

Third, given a bounded set $D \subseteq P$, we prove that both $\{T x: x \in D\}$ and $\left\{\Phi^{-1}(\rho(t))(T x)^{\prime}: x \in D\right\}$ are equicontinuous on each finite subinterval on $R$.

Then, there exists $r>0$ such that $D \subseteq\{x \in P:\|x\| \leq r\}$. Hence

$$
r=\max \left\{\sup _{t \in R}|x(t)|, \sup _{t \in R} \Phi^{-1}(\rho(t)) x^{\prime}(t)\right\}<+\infty .
$$

Then there exists a nonnegative function $\phi_{r} \in L^{1}(-\infty,+\infty)$ such that

$$
0 \leq f\left(t, x(t), x^{\prime}(t)\right)=f\left(t, x(t), \frac{1}{\Phi^{-1}(\rho(t))} \Phi^{-1}(\rho(t)) x^{\prime}(t)\right) \leq \phi_{r}(t), \quad t \in R .
$$

Then

$$
\left|A_{x}+\int_{t}^{+\infty} f\left(t, x(t), x^{\prime}(t)\right) d t\right| \leq \int_{-\infty}^{+\infty} \phi_{r}(t) d t:=L
$$


For any $\epsilon>0$, since $\Phi^{-1}$ is uniformly continuous on $[-L, L]$, there exists $\delta_{1}>0$ such that

$$
\left|\Phi^{-1}(u)-\Phi^{-1}(v)\right|<\epsilon, \quad u, v \in[-L, L],|u-v|<\delta_{1} .
$$

For any $a, b \in R, t_{1}, t_{2} \in[a, b]$ and $x \in D$ with $t_{1} \leq t_{2}$, we have

$$
\left|A_{x}+\int_{t_{1}}^{+\infty} f\left(t, x(t), x^{\prime}(t)\right) d t-A_{x}-\int_{t_{2}}^{+\infty} f\left(t, x(t), x^{\prime}(t)\right) d t\right| \leq \int_{t_{1}}^{t_{2}} \phi_{r}(s) d s .
$$

Then there exists $\delta>0$ such that

$$
\left|A_{x}+\int_{t_{1}}^{+\infty} f\left(t, x(t), x^{\prime}(t)\right) d t-A_{x}-\int_{t_{2}}^{+\infty} f\left(t, x(t), x^{\prime}(t)\right) d t\right|<\delta_{1}, \quad t_{1}, t_{2} \in[a, b],\left|t_{1}-t_{2}\right|<\delta .
$$

Hence $t_{1}, t_{2} \in[a, b],\left|t_{1}-t_{2}\right|<\delta$ imply that

$$
\begin{aligned}
& \left|\Phi^{-1}\left(\rho\left(t_{1}\right)\right)(T x)\left(t_{1}\right)-\Phi^{-1}\left(\rho\left(t_{2}\right)\right)(T x)\left(t_{2}\right)\right| \\
& \quad=\left|\Phi^{-1}\left(A_{x}+\int_{t_{1}}^{+\infty} f\left(t, x(t), x^{\prime}(t)\right) d t\right)-\Phi^{-1}\left(A_{x}+\int_{t_{2}}^{+\infty} f\left(t, x(t), x^{\prime}(t)\right) d t\right)\right|<\epsilon .
\end{aligned}
$$

It follows that $\{T x: x \in D\}$ is equicontinuous on each finite subinterval on $R$.

On the other hand, we have

$$
\begin{aligned}
\left|(T x)\left(t_{1}\right)-(T x)\left(t_{2}\right)\right| & =\int_{t_{1}}^{t_{2}} \Phi^{-1}\left(\frac{1}{\rho(s)}\right) \Phi^{-1}\left(A_{x}+\int_{s}^{+\infty} f\left(u, x(u), x^{\prime}(u)\right) d u\right) d s \\
& \leq \int_{t_{1}}^{t_{2}} \Phi^{-1}\left(\frac{1}{\rho(s)}\right) d s \Phi^{-1}(2 L) \\
& \longrightarrow 0 \text { uniformly as } t_{1} \longrightarrow t_{2} .
\end{aligned}
$$

Then $\left\{\Phi^{-1}(\rho(t))(T x)^{\prime}: x \in D\right\}$ is equicontinuous on each finite subinterval on $R$.

At last given a bounded set $D \subseteq P$, we show that both $\{T x: x \in D\}$ and $\left\{\Phi^{-1}(\rho(t))(T x)^{\prime}: x \in D\right\}$ are equiconvergent at $\pm \infty$, respectively.

Then, there exists $r>0$ such that $D \subseteq\{x \in P:\|x\| \leq r\}$. Hence

$$
r=\max \left\{\sup _{t \in R}|x(t)|, \sup _{t \in R} \Phi^{-1}(\rho(t)) x^{\prime}(t)\right\}<+\infty
$$


Then there exists a nonnegative function $\phi_{r} \in L^{1}(-\infty,+\infty)$ such that

$$
0 \leq f\left(t, x(t), x^{\prime}(t)\right)=f\left(t, x(t), \frac{1}{\Phi^{-1}(\rho(t))} \Phi^{-1}(\rho(t)) x^{\prime}(t)\right) \leq \phi_{r}(t), \quad t \in R .
$$

Then

$$
\left|A_{x}+\int_{t}^{+\infty} f\left(t, x(t), x^{\prime}(t)\right) d t\right| \leq \int_{-\infty}^{+\infty} \phi_{r}(t) d t:=L
$$

For any $\epsilon>0$, since $\Phi^{-1}$ is uniformly continuous on $[-L, L]$, there exists $\delta_{1}>0$ such that

$$
\left|\Phi^{-1}(u)-\Phi^{-1}(v)\right|<\epsilon, \quad u, v \in[-L, L],|u-v|<\delta_{1} .
$$

Since

$$
\left|\rho(t) \Phi\left((T x)^{\prime}(t)\right)-A_{x}\right|=\int_{t}^{\infty} f\left(u, x(u), x^{\prime}(u)\right) d u \leq \int_{t}^{\infty} \phi_{r}(u) d u \longrightarrow 0
$$

uniformly as $t \rightarrow \infty$, we get that there exists $T>0$ such that

$$
\left|\rho(t) \Phi\left((T x)^{\prime}(t)\right)-A_{x}\right|<\delta_{1}, \quad t \geq T
$$

Hence $t>T$ implies that

$$
\left|\Phi^{-1}(\rho(t))(T x)^{\prime}(t)-\Phi^{-1}\left(A_{x}\right)\right|=\left|\Phi^{-1}\left(\rho(t) \Phi\left((T x)^{\prime}(t)\right)\right)-\Phi^{-1}\left(A_{x}\right)\right|<\epsilon .
$$

Furthermore, we get

$$
\begin{aligned}
\mid(T x)(t) & -\frac{\int_{-\infty}^{+\infty} g(t) \int_{-\infty}^{t} \Phi^{-1}(1 / \rho(s)) \Phi^{-1}\left(A_{x}+\int_{s}^{+\infty} f\left(u, x(u), x^{\prime}(u)\right) d u\right) d s d t}{1-\int_{-\infty}^{+\infty} g(s) d s} \\
& +\int_{-\infty}^{+\infty} \Phi^{-1}\left(\frac{1}{\rho(s)}\right) \Phi^{-1}\left(A_{x}+\int_{s}^{+\infty} f\left(u, x(u), x^{\prime}(u)\right) d u\right) d s \mid \\
& =\int_{t}^{+\infty} \Phi^{-1}\left(\frac{1}{\rho(s)}\right) \Phi^{-1}\left(A_{x}+\int_{s}^{\infty} f\left(u, x(u), x^{\prime}(u)\right) d u\right) d s \\
& \leq \int_{t}^{+\infty} \Phi^{-1}\left(\frac{1}{\rho(s)}\right) d s \Phi^{-1}(2 L) \\
& \longrightarrow 0 \text { uniformly as } t \longrightarrow+\infty .
\end{aligned}
$$

Hence $\{T x: x \in D\}$ and $\left\{\Phi^{-1}(\rho(t))(T x)^{\prime}: x \in D\right\}$ are equiconvergent at $+\infty$. 
Similarly we can how that $\{T x: x \in D\}$ and $\left\{\Phi^{-1}(\rho(t))(T x)^{\prime}: x \in D\right\}$ are equiconvergent at $-\infty$. We omit the details.

Therefore, $T: P \rightarrow P$ is equiconvergent at $\pm \infty$. So the operator $T: P \rightarrow P$ is completely continuous.

Define the functionals on $P$ by

$$
\begin{gathered}
r(y)=\sup _{t \in R} \Phi^{-1}(\rho(t))\left|y^{\prime}(t)\right|, \quad y \in P, \\
\beta(y)=\sup _{t \in R}|y(t)|, \quad y \in P, \\
\theta(y)=\sup _{t \in R}|y(t)|, \quad y \in P, \\
\alpha(y)=\min _{t \in[-k, k]}|y(t)|, \quad y \in P, \\
\psi(y)=\min _{t \in[-k, k]}|y(t)|, \quad y \in P .
\end{gathered}
$$

It is easy to see that $\alpha, \psi$ are two nonnegative continuous concave functionals on the cone $P$, $\gamma, \beta, \theta$ are three nonnegative continuous convex functionals on the cone $P$ and $\alpha(y) \leq \beta(y)$ for all $y \in P$.

For $e_{1}, e_{2}, c>0$ and $1 \geq k>0$, define

$$
\begin{gathered}
M=\max \left\{\frac{\int_{-\infty}^{+\infty}\left(1 / \Phi^{-1}(\rho(s))\right) d s}{1-\int_{-\infty}^{+\infty} g(s) d s}, 1\right\}, \\
L_{1}=\int_{-k}^{0} \Phi^{-1}\left(\frac{1}{\rho(s)}\right) \Phi^{-1}(2 \sqrt{|s|}) d s \\
L_{2}=\int_{0}^{k} \Phi^{-1}\left(\frac{1}{\rho(s)}\right) \Phi^{-1}(2 \sqrt{s}) d s \\
W=\min \left\{\frac{\Phi(c)}{4+\pi}, \frac{\Phi(c)}{4+\pi} \Phi\left(\frac{1-\int_{-\infty}^{+\infty} g(s) d s}{\int_{-\infty}^{+\infty}\left(1 / \Phi^{-1}(\rho(s))\right) d s}\right)\right\} \\
W=\Phi\left(\frac{e_{2}}{\mu}\right) \max \left\{\Phi\left(\frac{1}{L_{1}}\right), \Phi\left(\frac{1}{L_{2}}\right)\right\}, \\
\delta(t)=\left\{\frac{\Phi^{2}\left(e_{1}\right)}{4+\pi}, \quad\left\{\frac{1}{t^{2}}, \quad|t| \geq 1\right.\right. \\
\mu=\frac{\int_{-\infty}^{-k} \Phi^{-1}(1 / \rho(s)) d s}{2 \int_{-\infty}^{+\infty} \Phi^{-1}(1 / \rho(s)) d s} .
\end{gathered}
$$


Theorem 2.8. Suppose that (H1)-(H3) hold. Given positive constants $e_{1}, e_{2}, c$ and $k \in(0,1)$, let $Q, W$, and $E$ be as above. If

$$
c \geq \frac{e_{2}}{\mu}>e_{2}>e_{1}>0, \quad Q \geq W
$$

and

(A1) $f\left(t, u,\left(1 / \Phi^{-1}(\rho(t))\right) v\right) \leq \delta(t) Q$ for all $t \in R, u \in[0, c], v \in[-c, c]$;

(A2) $f\left(t, u,\left(1 / \Phi^{-1}(\rho(t))\right) v\right) \geq \delta(t) W$ for all $t \in[-k, k], u \in\left[e_{2}, e_{2} / \mu\right], v \in[-c, c]$;

(A3) $f\left(t, u,\left(1 / \Phi^{-1}(\rho(t))\right) v\right) \leq \delta(t) E$ for all $t \in R, u \in\left[0, e_{1}\right], v \in[-c, c]$;

then $B V P(1.2)$ has at least three positive solutions $x_{1}, x_{2}, x_{3}$ such that

$$
\sup _{t \in R} x_{1}(t)<e_{1}, \quad \min _{t \in[-k, k]} x_{2}(t)>e_{2}, \quad \sup _{t \in R} x_{3}(t)>e_{1}, \quad \min _{t \in[-k, k]} x_{3}(t)<e_{2} .
$$

Proof. We prove that all conditions in Lemma 2.5 are satisfied.

(i) By the definitions, it is easy to show that $\alpha, \psi$ are two nonnegative continuous concave functionals on the cone $P, \gamma, \beta, \theta$ are three nonnegative continuous convex functionals on the cone $P$ and $\alpha(y) \leq \beta(y)$ for all $y \in P$. One sees $x \in P$ is a positive solution of BVP (1.2) if and only if $x$ is a solution of the operator equation $x=T x$.

(ii) For $y \in P$, we have

$$
\begin{aligned}
|y(t)| & =\left|\int_{-\infty}^{t} y^{\prime}(s) d s+y(-\infty)\right| \\
& \leq \int_{-\infty}^{t}\left|y^{\prime}(s)\right| d s+|y(-\infty)| \\
& \leq \int_{-\infty}^{t} \frac{1}{\Phi^{-1}(\rho(s))}\left|\Phi^{-1}(\rho(s)) y^{\prime}(s)\right| d s+\int_{-\infty}^{+\infty} g(s)|y(s)| d s \\
& \leq \int_{-\infty}^{+\infty} \frac{1}{\Phi^{-1}(\rho(s))} d s \sup _{t \in R} \Phi^{-1}(\rho(t))\left|y^{\prime}(t)\right| \int_{-\infty}^{+\infty} g(s) d s \sup _{t \in R}|y(t)| .
\end{aligned}
$$

It follows that

$$
\sup _{t \in R}|y(t)| \leq \int_{-\infty}^{+\infty} \frac{1}{\Phi^{-1}(\rho(s))} d s \sup _{t \in R} \Phi^{-1}(\rho(t))\left|y^{\prime}(t)\right| \int_{-\infty}^{+\infty} g(s) d s \sup _{t \in R}|y(t)| .
$$

Then

$$
\sup _{t \in R}|y(t)| \leq \frac{\int_{-\infty}^{+\infty}\left(1 / \Phi^{-1}(\rho(s))\right) d s}{1-\int_{-\infty}^{+\infty} g(s) d s} \sup _{t \in R} \Phi^{-1}(\rho(t))\left|y^{\prime}(t)\right|
$$


Hence

$$
\begin{aligned}
\|y\| & =\max \left\{\sup _{t \in R}|y(t)|, \sup _{t \in R} \Phi^{-1}(\rho(t))\left|y^{\prime}(t)\right|\right\} \\
& \leq \max \left\{\frac{\int_{-\infty}^{+\infty}\left(1 / \Phi^{-1}(\rho(s))\right) d s}{1-\int_{-\infty}^{+\infty} g(s) d s}, 1\right\} \sup _{t \in R} \Phi^{-1}(\rho(t))\left|y^{\prime}(t)\right| .
\end{aligned}
$$

It follows that $\|y\| \leq M \gamma(y)$ for all $y \in P$.

(iii) Corresponding to Lemma 2.5,

$$
c=c, \quad h=\mu e_{1}, \quad d=e_{1}, \quad a=e_{2}, \quad b=\frac{e_{2}}{\mu} .
$$

Now, we prove that all other conditions of Lemma 2.5 hold. One sees that $0<d<a$. The remainder is divided into five steps.

Step 1. Prove that $T: \overline{P_{c}} \rightarrow \overline{P_{c}} 0$.

For $y \in \overline{P_{c}}$, we have $\|y\| \leq c$. Then $0 \leq y(t) \leq c$ and $-c \leq \Phi^{-1}(\rho(t)) y^{\prime}(t) \leq c$ for all $t \in R$. So (A1) implies that

$$
f\left(t, y(t), y^{\prime}(t)\right)=f\left(t, y(t), \frac{1}{\Phi^{-1}(\rho(t))} \Phi^{-1}(\rho(t)) y^{\prime}(t)\right) \leq \delta(t) Q, \quad t \in R .
$$

We have

$$
\begin{aligned}
\Phi^{-1}(\rho(t))\left|(T y)^{\prime}(t)\right| & =\left|\Phi^{-1}\left(A_{y}+\int_{t}^{+\infty} f\left(u, y(u), y^{\prime}(u)\right) d u\right)\right| \\
& \leq \Phi^{-1}\left(\int_{-\infty}^{+\infty}\left|f\left(u, y(u), y^{\prime}(u)\right)\right| d u\right) \\
& \leq \Phi^{-1}\left(\int_{-\infty}^{+\infty} \delta(s) Q d r\right) \\
& =\Phi^{-1}(Q) \Phi^{-1}(4+\pi) \\
& \leq c .
\end{aligned}
$$

Similarly to (ii), we can show that

$$
\begin{aligned}
0 \leq(T y)(t) & \leq M \sup _{t \in R} \Phi^{-1}(\rho(t))\left|(T y)^{\prime}(t)\right| \\
& \leq M \Phi^{-1}\left(\int_{-\infty}^{+\infty} \delta(s) Q d r\right) \\
& =M \Phi^{-1}(Q) \Phi^{-1}(4+\pi) \\
& \leq c .
\end{aligned}
$$


It follows that

$$
\|T y\|=\max \left\{\sup _{t \in R}|(T y)(t)|, \sup _{t \in R} \Phi^{-1}(\rho(t))\left|(T y)^{\prime}(t)\right|\right\} \leq c
$$

Then $T: \overline{P_{c}} \rightarrow \overline{P_{c}}$.

Step 2. Prove that

$$
\{y \in P(\gamma, \theta, \alpha ; a, b, c) \mid \alpha(y)>a\}=\left\{y \in P\left(\gamma, \theta, \alpha ; e_{2}, \frac{e_{2}}{\mu}, c\right) \mid \alpha(y)>e_{2}\right\} \neq \emptyset
$$

and $\alpha(T y)>e_{2}$ for every $y \in P\left(\gamma, \theta, \alpha ; e_{2}, e_{2} / \mu, c\right)$.

Choose $y(t)=e_{2} / 2 \mu$ for all $t \in R$. Then $y \in P$ and

$$
\alpha(y)=\frac{e_{2}}{2 \mu}>e_{2}, \quad \theta(y)=\frac{e_{2}}{2 \mu} \leq \frac{e_{2}}{\mu}, \quad \gamma(y)=0<c .
$$

It follows that $\{y \in P(\gamma, \theta, \alpha ; a, b, c) \mid \alpha(y)>a\} \neq \emptyset$.

For $y \in P(\gamma, \theta, \alpha ; a, b, c)$, one has that

$$
\alpha(y)=\min _{t \in[-k, k]} y(t) \geq e_{2}, \quad \theta(y)=\sup _{t \in R} y(t) \leq \frac{e_{2}}{\mu}, \quad \gamma(y)=\sup _{t \in R}\left|y^{\prime}(t)\right| \leq c
$$

Then

$$
e_{2} \leq y(t) \leq \frac{e_{2}}{\mu}, \quad t \in[-k, k], \quad \Phi^{-1}(\rho(t))\left|y^{\prime}(t)\right| \leq c
$$

Thus (A2) implies that

$$
f\left(t, y(t), y^{\prime}(t)\right) \geq \delta(t) W, \quad t \in[-k, k]
$$

Similarly to Lemma 2.6(i), we know that there exists $\tau_{0} \in(0,1)$ such that $(T y)^{\prime}\left(\tau_{0}\right)=0$. Then

$$
(T y)(t)=\left\{\begin{array}{l}
(T y)(+\infty)+\int_{t}^{+\infty} \Phi^{-1}\left(\frac{1}{\rho(s)}\right) \Phi^{-1}\left(\int_{\tau_{0}}^{s} f\left(u, y(u), y^{\prime}(u)\right) d u\right) d s, \quad t \geq \tau_{0}, \\
(T y)(-\infty)+\int_{-\infty}^{t} \Phi^{-1}\left(\frac{1}{\rho(s)}\right) \Phi^{-1}\left(\int_{s}^{\tau_{0}} f\left(u, y(u), y^{\prime}(u)\right) d u\right) d s, \quad t \leq \tau_{0} .
\end{array}\right.
$$

Since

$$
\alpha(T y)=\min _{t \in[-k, k]}(T y)(t) \geq \mu \sup _{t \in R}(T y)(t)=\mu(T y)\left(\tau_{0}\right)
$$


if $\tau_{0} \geq 0$, we get

$$
\begin{aligned}
\alpha(T y) & \geq \mu\left[(T y)(-\infty)+\int_{-\infty}^{\tau_{0}} \Phi^{-1}\left(\frac{1}{\rho(s)}\right) \Phi^{-1}\left(\int_{s}^{\tau_{0}} f\left(u, y(u), y^{\prime}(u)\right) d u\right) d s\right] \\
& >\mu\left[\int_{-k}^{0} \Phi^{-1}\left(\frac{1}{\rho(s)}\right) \Phi^{-1}\left(\int_{s}^{0} f\left(u, y(u), y^{\prime}(u)\right) d u\right) d s\right] \\
& \geq \mu\left[\int_{-k}^{0} \Phi^{-1}\left(\frac{1}{\rho(s)}\right) \Phi^{-1}\left(\int_{s}^{0} \delta(u) W d u\right) d s\right] \\
& \geq \mu\left[\int_{-k}^{0} \Phi^{-1}\left(\frac{1}{\rho(s)}\right) \Phi^{-1}(2 \sqrt{|s|}) d s\right] \Phi^{-1}(W) \\
& \geq e_{2} .
\end{aligned}
$$

If $\tau_{0}<0$, we get

$$
\begin{aligned}
\alpha(T y) & \geq \mu\left[(T y)(+\infty)+\int_{\tau_{0}}^{+\infty} \Phi^{-1}\left(\frac{1}{\rho(s)}\right) \Phi^{-1}\left(\int_{\tau_{0}}^{s} f\left(u, y(u), y^{\prime}(u)\right) d u\right) d s\right] \\
& >\mu\left[\int_{\tau_{0}}^{k} \Phi^{-1}\left(\frac{1}{\rho(s)}\right) \Phi^{-1}\left(\int_{\tau_{0}}^{s} f\left(u, y(u), y^{\prime}(u)\right) d u\right) d s\right] \\
& \geq \mu\left[\int_{0}^{k} \Phi^{-1}\left(\frac{1}{\rho(s)}\right) \Phi^{-1}\left(\int_{0}^{s} \delta(u) W d u\right) d s\right] \\
& \geq \mu\left[\int_{0}^{k} \Phi^{-1}\left(\frac{1}{\rho(s)}\right) \Phi^{-1}(2 \sqrt{t}) d s\right] \Phi^{-1}(W) \\
& \geq e_{2} .
\end{aligned}
$$

This completes Step 2.

Step 3. Prove that $\{y \in Q(\gamma, \theta, \psi ; h, d, c) \mid \beta(y)<d\}=\left\{y \in Q\left(\gamma, \theta, \psi ; \mu e_{1}, e_{1}, c\right) \mid \beta(y)<e_{1}\right\} \neq \emptyset$ and

$$
\beta(T y)<e_{1} \quad \text { for every } y \in Q(\gamma, \theta, \psi ; h, d, c)=Q\left(\gamma, \theta, \psi ; \mu e_{1}, e_{1}, c\right)
$$

Choose $y(t)=\mu e_{1}$. Then $y \in P$, and

$$
\psi(y)=\mu e_{1} \geq h, \quad \beta(y)=\theta(y)=\mu e_{1}<e_{1}=d, \quad \gamma(y)=0 \leq c .
$$

It follows that $\{y \in Q(\gamma, \theta, \psi ; h, d, c) \mid \beta(y)<d\} \neq \emptyset$. 
For $y \in Q(\gamma, \theta, \psi ; h, d, c)$, one has that

$$
\psi(y)=\min _{t \in[-k, k]} y(t) \geq h=\mu e_{1}, \quad \theta(y)=\sup _{t \in R} y(t) \leq d=e_{1}, \quad \gamma(y)=\sup _{t \in R}\left|y^{\prime}(t)\right| \leq c .
$$

Hence we get that

$$
0 \leq y(t) \leq e_{1}, \quad t \in R ; \quad-c \leq \Phi^{-1}(\rho(t)) y^{\prime}(t) \leq c, \quad t \in R
$$

Then (A3) implies that

$$
f\left(t, y(t), y^{\prime}(t)\right) \leq \delta(t) E, \quad t \in R
$$

So

$$
\begin{aligned}
\beta(T y) & =M \sup _{t \in R} \Phi^{-1}(\rho(t))\left|(T y)^{\prime}(t)\right| \\
& \leq M\left|\Phi^{-1}\left(A_{y}+\int_{t}^{+\infty} f\left(u, y(u), y^{\prime}(u)\right) d u\right)\right| \\
& \leq M \Phi^{-1}\left(\int_{-\infty}^{+\infty}\left|f\left(u, y(u), y^{\prime}(u)\right)\right| d u\right) \\
& \leq M \Phi^{-1}\left(\int_{-\infty}^{+\infty} \delta(u) E d u\right) \\
& \leq M \Phi^{-1}(4+\pi) \Phi^{-1}(E) \\
& =e_{1}=d .
\end{aligned}
$$

This completes Step 3.

Step 4. Prove that $\alpha(T y)>a$ for $y \in P(\gamma, \alpha ; a, c)$ with $\theta(T y)>b$;

For $y \in P(\gamma, \alpha ; a, c)=P\left(\gamma, \alpha ; e_{2}, c\right)$ with $\theta(T y)>b=e_{2} / \mu$, we have that $\alpha(y)=$ $\min _{t \in[-k, k]} y(t) \geq e_{2}$ and $\gamma(y)=\sup _{t \in R} \Phi^{-1}(\rho(t))\left|y^{\prime}(t)\right| \leq c$ and $\sup _{t \in R}(T y)(t)>e_{2} / \mu$. Then

$$
\alpha(T y)=\min _{t \in[-k, k]}(T y)(t) \geq \mu \beta(T y)>\mu \frac{e_{2}}{\mu}=e_{2}=a
$$

This completes Step 4.

Step 5. Prove that $\beta(T y)<d$ for each $y \in Q(\gamma, \beta ; d, c)$ with $\psi(T y)<h$.

For $y \in Q(\gamma, \beta ; d, c)$ with $\psi(T y)<d$, we have $\gamma(y)=\sup _{t \in R} \Phi^{-1}(\rho(t))\left|y^{\prime}(t)\right| \leq c$ and $\beta(y)=\sup _{t \in R} y(t) \leq d=e_{1}$ and $\psi(T y)=\min _{t \in[-k, k]}(T y)(t)<h=e_{1} \mu$. Then

$$
\beta(T y)=\sup _{t \in R}(T y)(t) \leq \frac{1}{\mu} \min _{t \in[-k, k]}(T y)(t)<\frac{1}{\mu} e_{1} \mu=e_{1}=d
$$

This completes the Step 5 . 
Then Lemma 2.5 implies that $T$ has at least three fixed points $x_{1}, x_{2}$, and $x_{3}$ such that

$$
\beta\left(x_{1}\right)<e_{1}, \quad \alpha\left(x_{2}\right)>e_{2}, \quad \beta\left(x_{3}\right)>e_{1}, \quad \alpha\left(x_{3}\right)<e_{2} .
$$

Hence BVP(1.2) has three decreasing positive solutions $x_{1}, x_{2}$ and $x_{3}$ such that (2.70) holds. The proof is complete.

\section{Examples}

Now, we present an example, whose three positive solutions cannot be obtained by theorems in known papers, to illustrate the main results.

Example 3.1. Consider the following BVP

$$
\begin{aligned}
& {\left[e^{3 t^{2}}\left(x^{\prime}(t)\right)^{3}\right]^{\prime}+f\left(t, x(t), x^{\prime}(t)\right)=0, \quad t \in R,} \\
& x(-\infty)=0, \\
& x(+\infty)=0 .
\end{aligned}
$$

Corresponding to BVP(1.2), one sees that $\phi(x)=x^{3}, \phi^{-1}(x)=x^{1 / 3}, g(t)=h(t) \equiv 0, \rho(t)=e^{3 t^{2}}$, $f: R \times R \times R \rightarrow[0, \infty)$ is nonnegative and continuous and is defined by

$$
\begin{gathered}
f(t, x, y)=\delta(t)\left(f_{0}(x)+g_{0}\left(e^{t^{2}}|y|\right)\right), \\
\delta(t)= \begin{cases}\frac{1}{t^{2}}, & |t| \geq 1, \\
\frac{1}{\sqrt{|t|}}, & |t| \leq 1 .\end{cases}
\end{gathered}
$$

Choose $k=1, e_{1}=50, e_{2}=250, c=40000$. By direct computation, we see that $Q, W$, and $E$ are given by

$$
\begin{aligned}
& M=\max \left\{\frac{\int_{-\infty}^{+\infty}\left(1 / \Phi^{-1}(\rho(s))\right) d s}{1-\int_{-\infty}^{+\infty} g(s) d s}, 1\right\}=\frac{\sqrt{\pi}}{2} \\
& L_{1}=\int_{-k}^{0} \Phi^{-1}\left(\frac{1}{\rho(s)}\right) \Phi^{-1}(2 \sqrt{|s|}) d s=2^{1 / 3} \int_{-1}^{0} e^{-s^{2}}|s|^{1 / 6} d s>0.06 \\
& L_{2}=\int_{0}^{k} \Phi^{-1}\left(\frac{1}{\rho(s)}\right) \Phi^{-1}(2 \sqrt{s}) d s=2^{1 / 3} \int_{0}^{1} e^{-s^{2}} s^{1 / 6} d s>0.06
\end{aligned}
$$




$$
\begin{aligned}
\mu & =\frac{\int_{-\infty}^{-k} \Phi^{-1}(1 / \rho(s)) d s}{2 \int_{-\infty}^{+\infty} \Phi^{-1}(1 / \rho(s)) d s}=\frac{\int_{-\infty}^{-1} e^{-s^{2}} d s}{\sqrt{\pi}}>0.37 \\
Q & =\min \left\{\frac{\Phi(c)}{4+\pi}, \frac{\Phi(c)}{4+\pi} \Phi\left(\frac{1-\int_{-\infty}^{+\infty} g(s) d s}{\int_{-\infty}^{+\infty}\left(1 / \Phi^{-1}(\rho(s))\right) d s}\right)\right\}=\frac{64 \times 10^{12}}{4+\pi}>8.53 \times 10^{12}, \\
W & =\Phi\left(\frac{e_{2}}{\mu}\right) \max \left\{\Phi\left(\frac{1}{L_{1}}\right), \Phi\left(\frac{1}{L_{2}}\right)\right\}<16.67^{3} \times 675.7^{3}<3.43 \times 10^{11}, \\
E & =\frac{\Phi\left(e_{1}\right)}{4+\pi}=\frac{2500}{4+\pi}>333.33 .
\end{aligned}
$$

One can show that

$$
c \geq \frac{e_{2}}{\mu}>e_{2}>e_{1}>0, \quad Q \geq W .
$$

Suppose that

$$
\begin{gathered}
f_{0}(x)= \begin{cases}166.67, & x \in[0,50], \\
166.67+\frac{44.36 \times 10^{11}-166.67}{250-50}(x-50), & x \in[50,250], \\
44.36 \times 10^{11}, & x \in[250,40000], \\
44.36 \times 10^{11} e^{x-40000,} & x \geq 40000,\end{cases} \\
\qquad g_{0}(y) \mid \leq 10, \quad \forall y \in R .
\end{gathered}
$$

From

$$
f\left(t, x, e^{-t^{2}} y\right)=\delta(t)\left[f_{0}(x)+g_{0}(y)\right]
$$

it is easy to show that
$\left(\mathrm{A}_{1}\right) f\left(t, u, e^{-t^{2}} v\right) \leq 8.53 \times 10^{12} \delta(t)$ for all $t \in R, u \in[0,400000], v \in[-40000,40000]$;
$\left(\mathrm{A}_{2}\right) f\left(t, u, e^{-t^{2}} v\right) \geq 3.43 \times 10^{11} \delta(t)$ for all $t \in[-1,1], u \in[250,1000], v \in[-40000,40000]$;
$\left(\mathrm{A}_{3}\right) f\left(t, u, e^{-t^{2}} v\right) \leq 333.33 \delta(t)$ for all $t \in R, u \in[0,50], v \in[-40000,40000]$;

then Theorem 2.8 implies that BVP(3.1) has at least three positive solutions $x_{1}, x_{2}, x_{3}$ such that

$$
\begin{array}{ll}
\sup _{t \in R} x_{1}(t)<50, & \min _{t \in[-1,1]} x_{2}(t)>250, \\
\sup _{t \in R} x_{3}(t)>50, & \min _{t \in[-1,1]} x_{3}(t)<250 .
\end{array}
$$

Remark 3.2. Example 3.1 implies that there is a large number of functions that satisfy the conditions of Theorem 2.8. In addition, the conditions of Theorem 2.8 are also easy to check. 


\section{Acknowledgments}

This work was supported by Natural Science Foundation of Guangdong provinces Grant (no S2011010001900) and the Foundation for High-level talents in Guangdong Higher Education Project.

\section{References}

[1] V. A. Il'in and E. I. Moiseev, "Nonlocal boundary-value problem of the second kind for a SturmLiouville operator," Differential Equations, vol. 23, pp. 979-987, 1987.

[2] R. P. Agarwal, Boundary Value Problems for Higher Order Differential Equations, World Scientific, Singapore, 1986.

[3] K. Deimling, Nonlinear Functional Analysis, Springer, Berlin, Germany, 1985.

[4] W. Ge, Boundary Value Problems for Ordinary Differential Equations, Science Press, Beijing, China, 2007.

[5] G. Cupini, C. Marcelli, and F. Papalini, "Heteroclinic solutions of boundary-value problems on the real line involving general nonlinear differential operators," Differential and Integral Equations, vol. 24, no. 7-8, pp. 619-644, 2011.

[6] C. Marcelli and F. Papalini, "Heteroclinic connections for fully non-linear non-autonomous secondorder differential equations," Journal of Differential Equations, vol. 241, no. 1, pp. 160-183, 2007.

[7] A. Cabada and J. A. Cid, "Heteroclinic solutions for non-autonomous boundary value problems with singular $\Phi$-Laplacian operators, discrete and continuous dynamical systems," in Proceedings of the 7th AIMS International Conference on Dynamical Systems, Differential Equations and Applications, pp. 118-122, 2009.

[8] A. Calamai, "Heteroclinic solutions of boundary value problems on the real line involving singular Ф-Laplacian operators," Journal of Mathematical Analysis and Applications, vol. 378, no. 2, pp. 667-679, 2011.

[9] G. Cupini, C. Marcelli, and F. Papalini, "On the solvability of a boundary value problem on the real line," Boundary Value Problems, vol. 2011, article 26, 2011.

[10] R. I. Avery, "A generalization of the Leggett-Williams fixed point theorem," Mathematical Sciences Research Hot-Line, vol. 2, no. 7, pp. 9-14, 1999. 


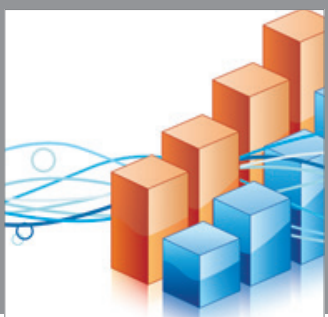

Advances in

Operations Research

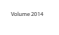

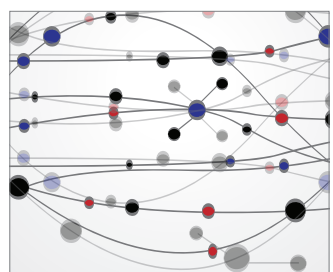

\section{The Scientific} World Journal
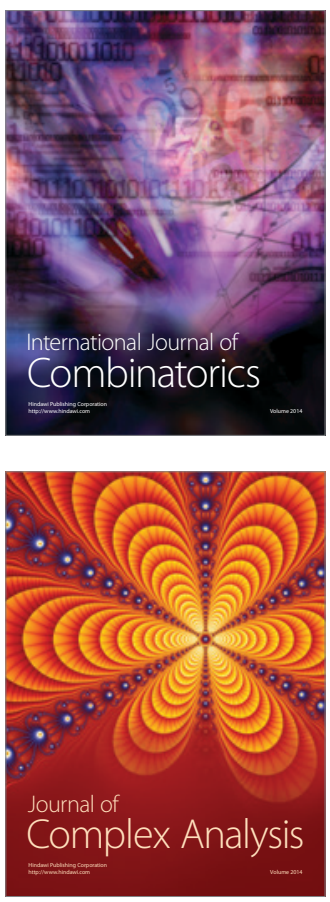

International Journal of

Mathematics and

Mathematical

Sciences
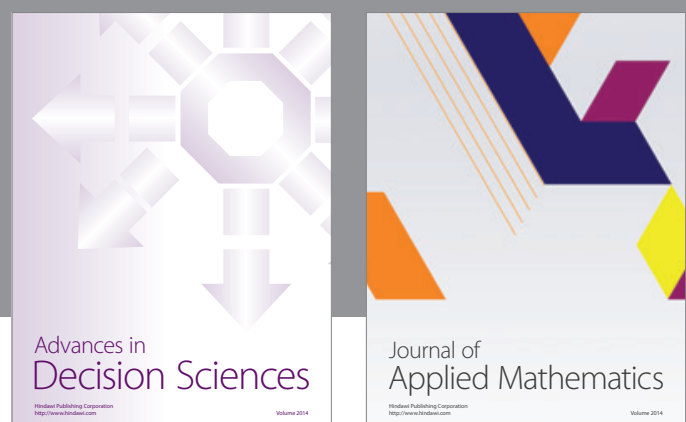

Journal of

Applied Mathematics
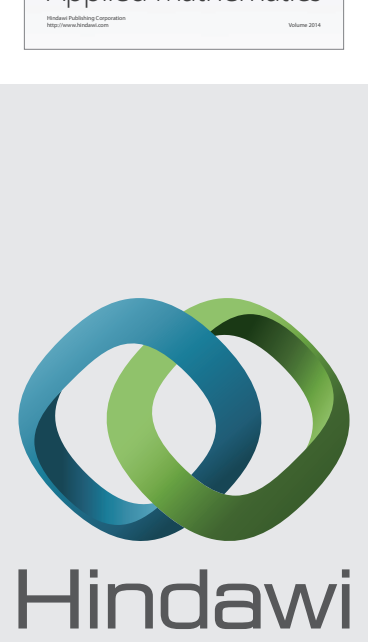

Submit your manuscripts at http://www.hindawi.com
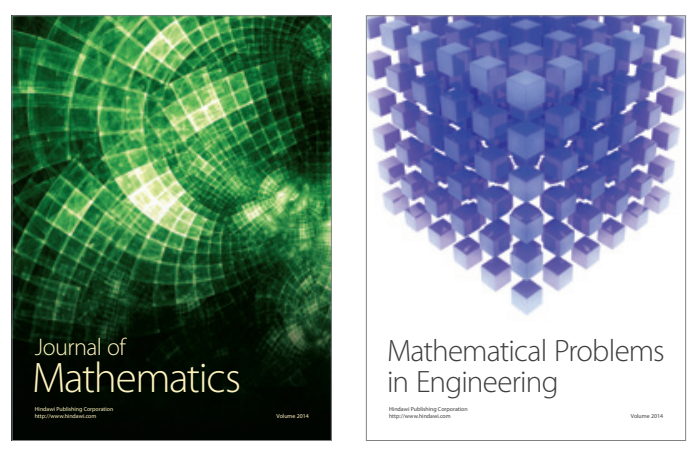

Mathematical Problems in Engineering
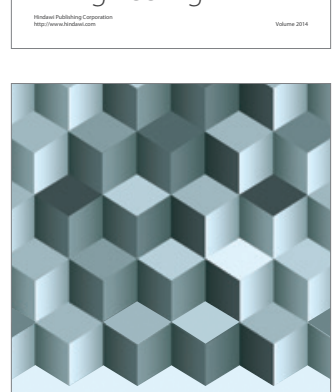

Journal of

Function Spaces
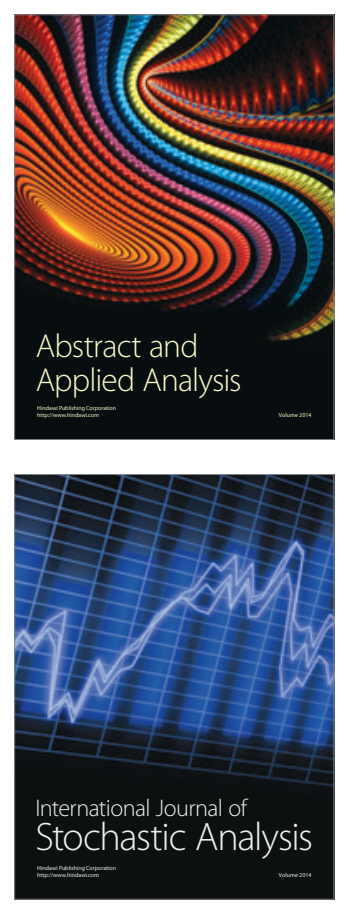

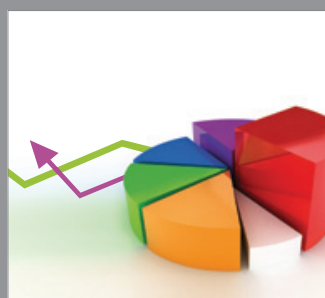

ournal of

Probability and Statistics

Promensencen
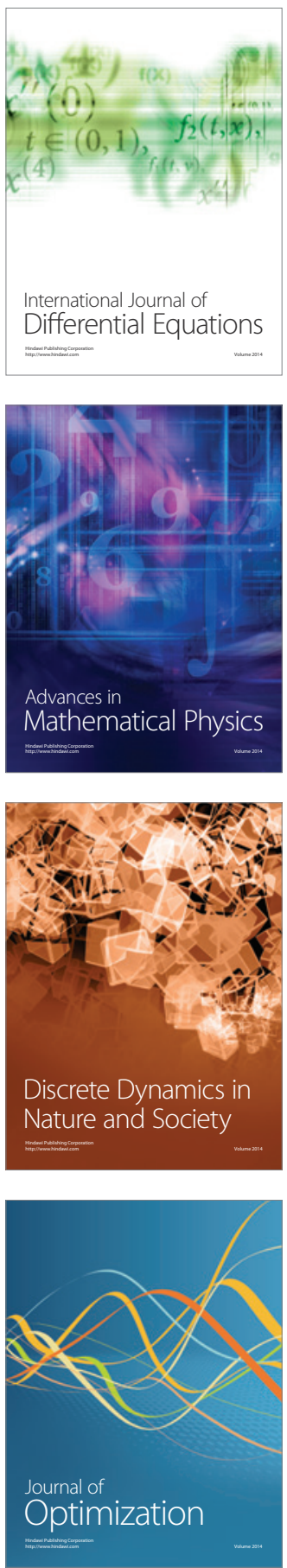